\title{
Micro-Mechanical Investigation of Low Temperature Fatigue Cracking Behaviour of Bitumen
}

\author{
Prabir Kumar Das, Denis Jelagin, Björn Birgisson and Niki Kringos \\ Division of Highway and Railway Engineering, Transport Science \\ Department, KTH Royal Institute of Technology, Sweden
}

\begin{abstract}
In an effort to understand the effect of low temperature fatigue cracking, atomic force microscopy (AFM) was used to characterize the morphology of bitumen. In addition, thermal analysis and chemical characterization was done using differential scanning calorimetry (DSC) and thin-layer chromatography / flame ionization detection (TLC/FID), respectively. The AFM topographic and phase contrast image confirmed the existence of bee-shaped microstructure and different phases. The bitumen samples were subjected to both environmental and mechanical loading and after loading, micro-cracks appeared in the interfaces of the bitumen surface, confirming bitumen itself may also crack. It was also found that the presence of wax and wax crystallization plays a vital role in low temperature cracking performance of bitumen.
\end{abstract}

\section{Introduction}

Low temperature cracking is known to be one of the major distresses that result in both structural and functional problems for asphalt pavements. When the temperature decreases, asphalt mixtures tend to contract which results in an increase in tensile stresses inside the pavement. If these tensile stresses exceed the tensile strength of the asphalt mixture, cracks may start propagating inside the material and this often results in visible transverse cracks on the pavement surface [1]. These cracks may then lead to other types of pavement deteriorations, since water can now freely access the structure and traffic loading may give added local impact forces. Cracking is therefore a significant contributor to the overall reduction of the pavement service life and an increase in maintenance cost.

There are, of course, many different causes for cracks to initiate and propagate in an asphaltic pavement. Even on top of a rut, which is caused at higher temperatures, often a crack appears. Nevertheless, cracking may develop due to a 
critical low ambient air temperature drop which is known as low temperature cracking or due to several thermal cycles which is known as thermal fatigue cracking. This paper is mainly focused on thermal fatigue cracking. Asphalt mixtures consist of a matrix of bitumen and mineral aggregates where each component has its own stiffness, strength and contraction-expansion rates. Because of this change in properties, cracks may develop in the interfaces between the aggregate and bitumen, as shown in Figure 1 (a). The existence of microstructures in bitumen matrix found in several studies proves the heterogeneity of bitumen [24]. This inhomogeneity is creating internal interfaces within the bitumen matrix. Bitumen have therefore zones that, just like mineral-bitumen interfaces, have a tendency to act as natural stress inducers. Thus, at low temperatures when the bitumen becomes stiff, this induced stress may cause cracking which could propagate through bitumen, as shown in Figure 1 (b).

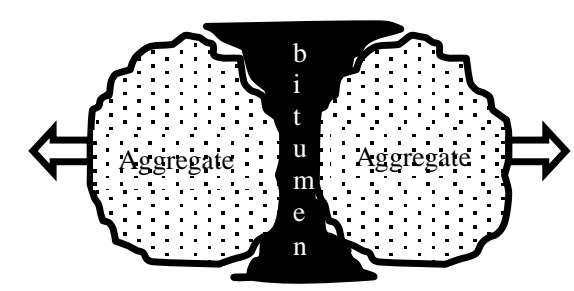

(a)

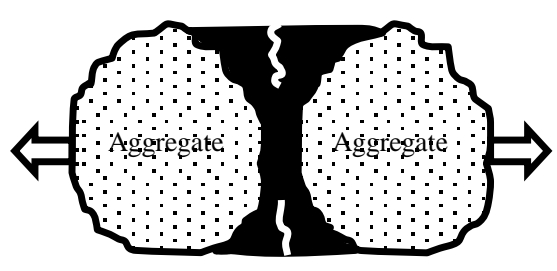

(b)

Figure 1. Two possible ways of crack initiation due to thermal cracking: a) interface and b) through bitumen.

Over many years, a great number of efforts have been made to investigate the complexity of the thermal fatigue problem of asphalt pavements. Models for predicting low temperature cracking have also been proposed. Nevertheless, until now a test method is not available to investigate mastic (i.e., bitumen and fillers) and bitumen resistance to thermal fatigue. Also, most of the existing specifications and theories do not address cracking of bitumen itself due to thermal fatigue. Yet, according to many researchers, bitumen is an important component that needs to be considered when investing low temperature or thermal fatigue cracking of asphalt mixtures [5-6].

For this reason, the present study is focusing on a micro-mechanical investigation of the thermal fatigue cracking behaviour of the bitumen itself, where the hypothesis is that bitumen itself may also crack due to its inherent the heterogeneities under environmental and traffic loading. 


\section{Low temperature properties of bitumen}

Bitumen is a thermoplastic material that at high temperatures has a very low and at low temperatures has a very high viscosity. Initially when a pavement cools, the bitumen is viscous enough to shrink due to its stress relaxation behaviour. The bitumen stiffens upon further cooling and reaches a certain temperature at which the thermal stress can no longer relax through this viscous mechanism. This phenomenon also occurs with bitumen when subjected to several thermal cycles. From a chemical point of view at lower temperatures the fluid non-polar molecules begin to organize themselves into a structured form. Since bitumen contains both polar and non-polar components, at low temperatures these non-polar molecules combine with the already-structured polar molecules and make asphalt more rigid and likely to fracture when exposed to stresses.

In addition to this phenomenon, waxes are also often an important component to consider in the thermal fatigue behaviour of bitumen, even though its behaviour is not fully understood to date. Natural waxes may be present at all times at various concentration in bitumen, depending on the crude source type and refinery process, and sometimes commercial waxes are added into bitumen or asphalt mixtures to allow for sufficient flow when reducing the mixing and compaction temperature [7]. Wax modification may also have a positive healing effect on bitumen, thus improving the long-term performance of asphalt pavements [8-9]. Crystallizing wax in bitumen may have negative effect on bitumen properties and may increase the sensitivity to plastic deformation or cracking in asphalt pavement. Thus wax crystallization and melting in bitumen is considered an important issue when it comes to quality and performance [10].

\section{The importance of understanding the fundamental behaviour}

Thermorheological behaviour of bitumen is an important factor for understanding the performance-based optimization of asphalt mixture. The temperature dependent rheological behaviour of bitumen depends on the chemical structure and intermolecular associations (structuring) [4]. This structuring is largely responsible for the physical properties of bitumen thus the prediction of the performance of asphalt pavements should also directly be related to this [2, 11]. Structuring may occur at various ranges from molecular to macroscopic but most of the asphalt researchers have concerned themselves with the microstructures of the bitumen itself. Bitumen is a complex mixture of molecules of different size and polarity, for which microstructural knowledge is still rather incomplete. Once we have knowledge at that level, by upscaling material parameters from microscale observations, a multiscale model can be proposed which will allow us to predict cracks in the pavement, based on the fundamental knowledge on smaller sales.

From extensive atomic force microscopy (AFM) investigations shown in earlier papers [2-4, 11-14], it has been shown that bitumen has the tendency to phase 
separate under certain kinetic conditions, leading to a predominant clustering of two types of phases, illustrated in Figure 2. To ensure that the found phenomena of phase separation in bitumen is not a side effect of sample preparation or a surface effect in the AFM, several other experiments were also performed, among which an extensive neutron scattering study [12]. From mechanical considerations, it is known that the interfaces between two materials with different stiffness properties serve as natural stress inducers. This means that when the material is exposed to mechanical and or environmental loading, these interfaces will attract high stresses and are prone to cracking.
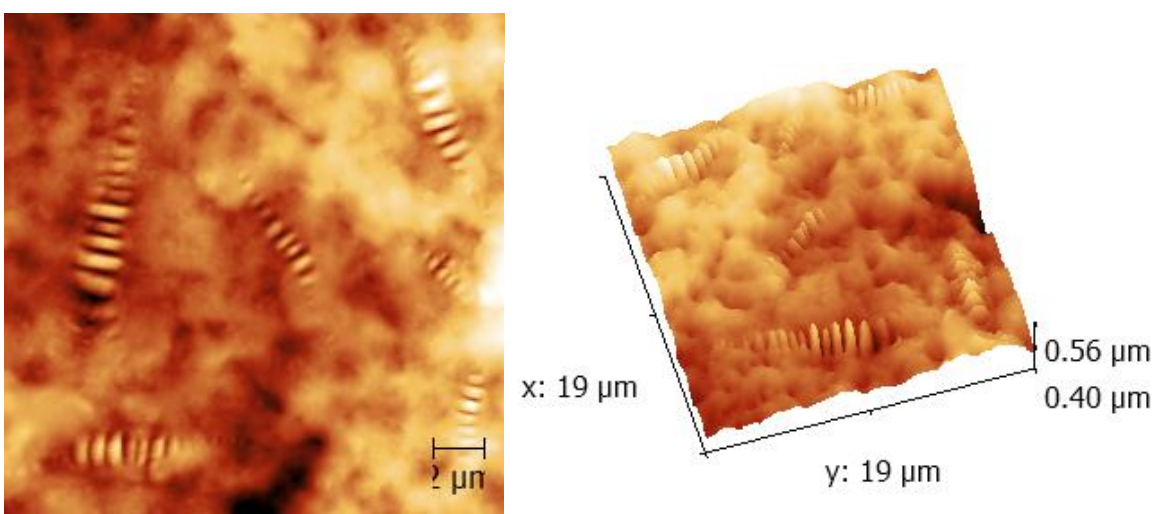

Figure 2. Topographic 2D (left) and 3D right AFM image $(19 \mu \mathrm{m} \times 19 \mu \mathrm{m})$ of bitumen indicating evidence of microstructures.

Thus, it would be of great interest to understanding the conditions under which this phase behaviour occurs and the speed, or mobility, of this microstructure appearance. For this reason, this paper is presenting preliminary results of an ongoing project to characterize bitumen behaviour on multiple scales.

\section{Experimental Study:}

\section{Material}

An unaged bitumen was used in this study provided by Nynan Bitumen, Sweden. The physical-chemical properties of this bitumen are presented in Table I. The penetration and softening point were measured in accordance with the European standards EN 1426 and EN 1427, respectively. The chemical characterization (SARA analysis) of bitumen was done by using the thin-layer chromatography with flame ionization detection (TLC-FID) of which the detail procedure can be found in a previous study [15]. 
Table I. Physical chemical properties of bitumen

\begin{tabular}{lll}
\hline & Characteristics & Result \\
\hline Physical properties & Penetration $\left(25^{\circ} \mathrm{C}, 0.1 \mathrm{~mm}\right)$ & 86 \\
& Softening point $\left({ }^{\circ} \mathrm{C}\right)$ & 46.4 \\
\hline Chemical components & Saturates $(\%)$ & 11 \\
& Aromatics $(\%)$ & 55 \\
& Resins (\%) & 19 \\
& Asphaltenes (\%) & 15 \\
\hline
\end{tabular}

\section{Atomic Force Microscopy (AFM)}

As described in earlier section, a detailed knowledge of microstructure is needed to understand the physico-chemistry of bitumen, which can serve as the direct link between the molecular structure and the rheological behaviour. Optical microscopy techniques have been employed to have a better understanding and visualization of bitumen microstructures [10]. However, because of the opacity and adhesive properties of bitumen, optical microscopy has not received much attention from the asphalt industry. To overcome some of the limitations of optical microscopy, researcher in the asphalt field have chosen to use scanning probe microscopy such as the AFM. AFM is capable of measuring topographic features at atomic and molecular resolutions as compared to the resolution limit of optical microscopy of about 200nm. Moreover, AFM has the advantage of imaging almost any type of surface which opens the window for investigating micro-cracks due to thermal fatigue.

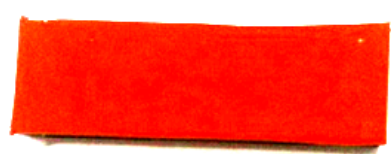

(a)

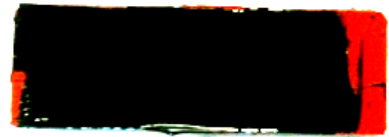

(b)

Figure 3. (a) Silicon bar of $60 \mathrm{~mm} \times 20 \mathrm{~mm} \times 7 \mathrm{~mm}(\mathrm{~b})$ bitumen film on top of it

Sample preparation: In this study, AFM (using a VECO Dimension 3100 device) was used for the microstructural characterization of the investigated bitumen. Approximately $30 \mathrm{mg}$ of hot bitumen was carefully placed on a rectangular silicon bar $(60 \mathrm{~mm} \times 20 \mathrm{~mm} \times 7 \mathrm{~mm})$ and it was spread out with a blade to form a relatively thin film, as shown in Figure 3. Then the bitumen film was cooled to room temperature and covered to prevent dust pick-up. After that the sample was annealed at $25^{\circ} \mathrm{C}$ for a minimum of $24 \mathrm{~h}$ before imaging [13]. The scanning was 
carried out in tapping mode at room temperature $\left(25^{\circ} \mathrm{C}\right)$ and scan rate was $1 \mathrm{~Hz}$. With Veeco Nanoprobe TM cantilevers with spring constant $40 \mathrm{~N} / \mathrm{m}$ and resonant frequency $300 \mathrm{kHz}$. AFM images were acquired at several locations on the sample surface to have complete information of the bitumen sample.

\section{Thermal fatigue procedure}

The bitumen film over the silicon bar was then subjected to thermal fatigue with heating and cooling cycles. A freezer was used to regulate the low temperature at $20^{\circ} \mathrm{C}$ and a room with controlled temperature at $25^{\circ} \mathrm{C}$ was used for thawing. The experimental method of thermal fatigue cycles (each cycle took 40 minutes) in this study is explained in the Figure 4. In between each thermal cycle the sample was subjected to additional tensile stress. This was done by bending at the mid-point of the silicon bar at a controlled angle of 3 degrees. The sample underwent 15 cycles of thermal loading; after the last freezing, the sample was placed under the AFM to investigate change in micro-structure due to the thermal fatigue.

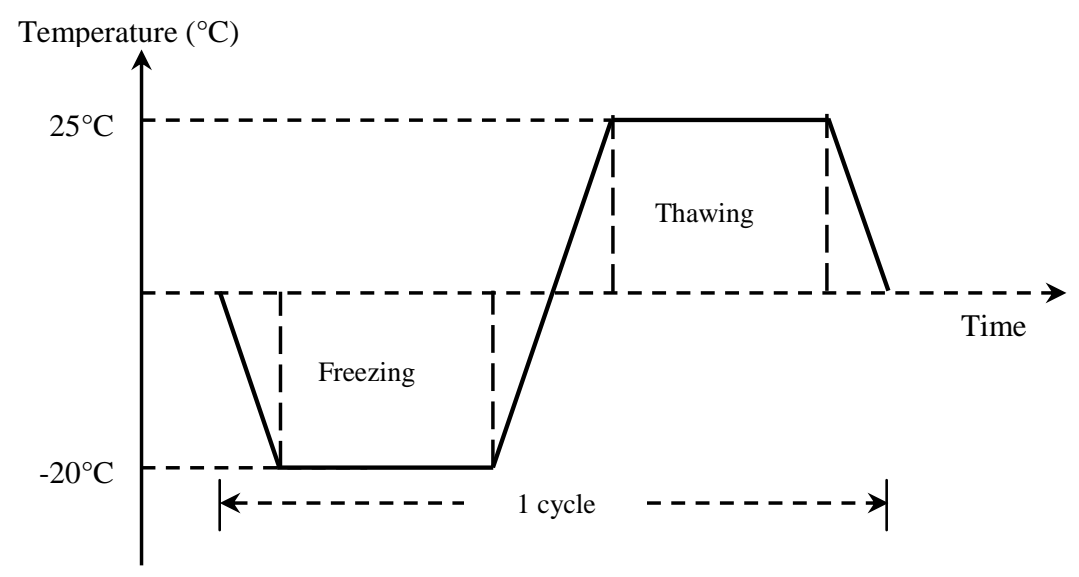

Figure 4. Thermal loading cycle

\section{Differential Scanning Calorimetry (DSC)}

DSC analysis was conducted to obtain the heat flow characteristics of bitumen. The tests were performed using a TA instrument (model TA Q2000) equipped with a refrigerated cooling system. Approximately $15 \mathrm{mg}$ of bitumen was scraped from the sample container and hermetically sealed into DSC sample pan. The sample pan was then placed horizontally on a hot plate at $80^{\circ} \mathrm{C}$ for 15 minutes to let the bitumen touch the pan bottom and also level out the surface. After that the sample was annealed at $25^{\circ} \mathrm{C}$ for a minimum of $24 \mathrm{~h}$. The sample was cooled from $25^{\circ} \mathrm{C}$ to $-80^{\circ} \mathrm{C}$ and kept at this temperature for $5 \mathrm{~min}$. Data was recorded during heating from $-80^{\circ} \mathrm{C}$ to $+90^{\circ} \mathrm{C}$ and cooling from $+90^{\circ} \mathrm{C}$ to $-80^{\circ} \mathrm{C}$. Finally, a second heating 
was done from $-80^{\circ} \mathrm{C}$ to $+90^{\circ} \mathrm{C}$ and data was recorded. In total, for each sample one cooling and two heating scans data was recorded. The heating and cooling rate was $10^{\circ} \mathrm{C} / \mathrm{min}$. Wax content was determined from endothermic pick during the heating scan and while calculation a constant melting enthalpy of $121 \mathrm{~J} / \mathrm{g}$ was used as reference [10].

\section{Results and discussions}

\section{Basic morphology}

The basic morphology of the bitumen was investigated in a clean room environment with a controlled temperature. Typical topographic and phase contrast image of the bitumen surface obtained at $25^{\circ} \mathrm{C}$ are presented in Figure 5, where one can easily observe the existing of phase separation in the bitumen matrix as reported earlier by several researchers [2-4, 11-14]. The rippled microstructures are also observed which are often referred to as bee-structures. The pale and dark lines indicate rise and drop of the topographic profile against the background, which are also known as peaks and valleys, respectively. A phase shift less than $10^{\circ}$ can be detected between the bright and darker network. The shades in the phase contrast images indicate the relative stiffness of the phases as obtained from sample-tip interaction. The greater sample-tip interaction results softer phase and dark shade in the phase image. Thus, the evidence of different stiffness in the bitumen matrix can also be observed.
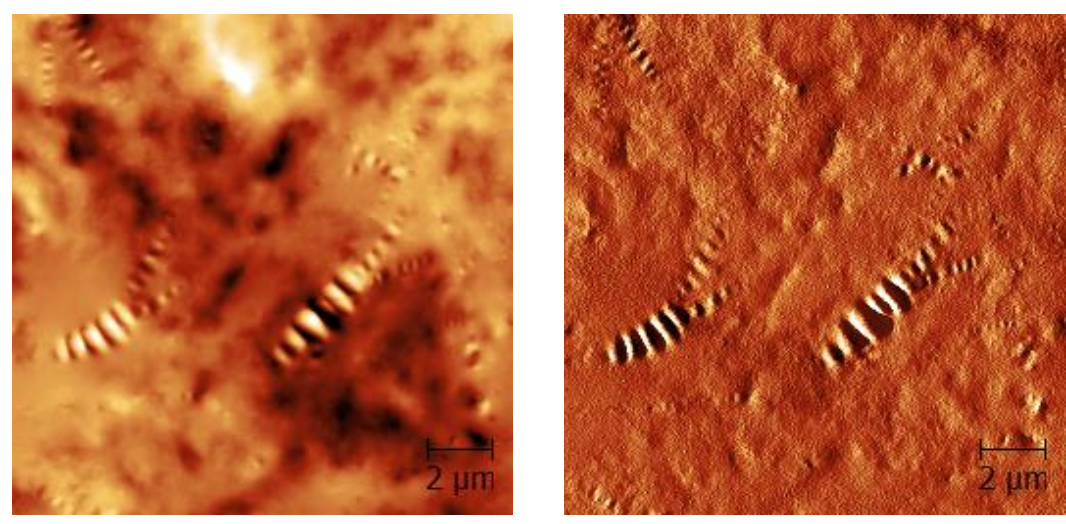

Figure 5. AFM topographic (left) and phase contrast (right) images of bitumen at $25^{\circ} \mathrm{C}(15 \mu \mathrm{m} \times 15 \mu \mathrm{m})$.

All of the previous research [2-4, 11-14] on bitumen under AFM mentioned the so called bee-shaped structures, thus it would be a great interest to zoom into bees to have a clear idea. Figure 6 shows a line profile along a bee which illustrates the 
earlier mentioned higher and lower parts of the bee-shaped structures. The mean topographical change and the distance between higher parts of bees are recorded as $68 \mathrm{~nm}$ and $567 \mathrm{~nm}$, respectively. These measured values are consistent to those reported by Loeber et al. (1996), Jäger et al. (2004) and Masson et al. (2007) $[2,3,14]$.
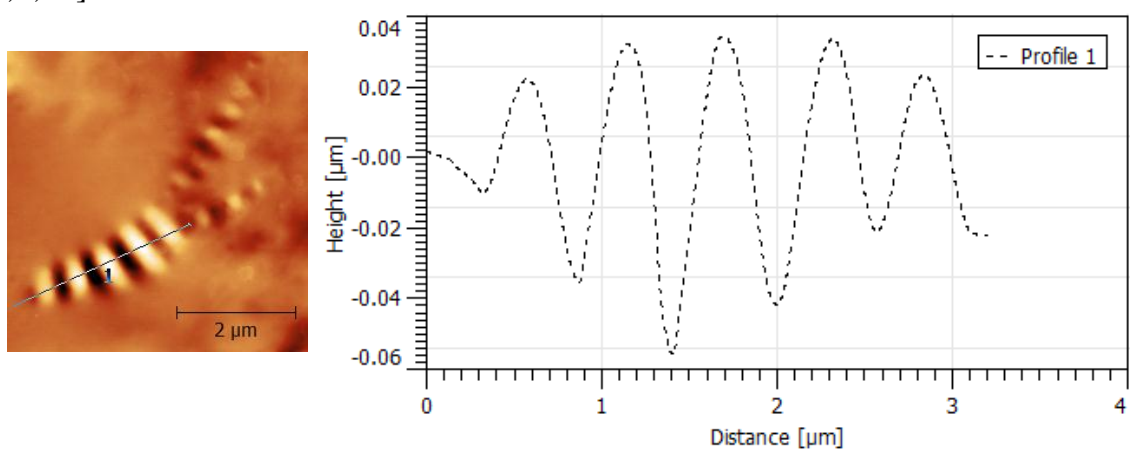

Figure 6. Topographic image $(5 \mu \mathrm{m} \times 5 \mu \mathrm{m})$ and a line profile along bee-shaped structure

\section{DSC Characterisation}

DSC has widely applied to determine glass transition temperature, wax content, wax crystallisation starting and melting out temperature of bituminous materials. In the case of bitumen, the interpretation of DSC curve is rather complicated since there are several overlapping phenomena to consider. Bitumen is a complex mixture of different molecules thus the glass transition phenomenon of bitumen occurs over a large temperature range. It can be seen from heat flow diagram (cf. Figure 7), the glass transition stated at around $-40^{\circ} \mathrm{C}$ and followed by a weak exothermic effect around $-10^{\circ} \mathrm{C}$ caused by cold crystallization of wax which could not crystallize through the cooling cycle due to limited mobility. In the cooling cycle, an exothermic transition occurs at around $+50^{\circ} \mathrm{C}$ which usually is interpreted as starting of crystallisation. It can be also found that the crystallization takes place during a range of temperatures, indicating the presence of molecules with different crystallization points. At around $+40^{\circ} \mathrm{C}$ to $+60^{\circ} \mathrm{C}$ the wax completely melted. The wax content according to DSC was found $6.2 \%$.

According to Pauli et al. (2011) [4], this wax crystallization is influencing for much of the development and appearance of microstructures, including the wellknown bee-structures (cf. Figure 5). In addition, paraffin waxes could exhibit crystalline forms at ambient temperature, which could also responsible for microstructuring. Thus, the appearance of surface structuring is related to the wax types and concentrations. It is important to consider wax crystallization of bitumen used in asphalt pavement because it occurs in a temperature range that coincides with the pavement service temperature. Moreover, it is highly probable that wax crystallization affect low temperature properties of bitumen. 


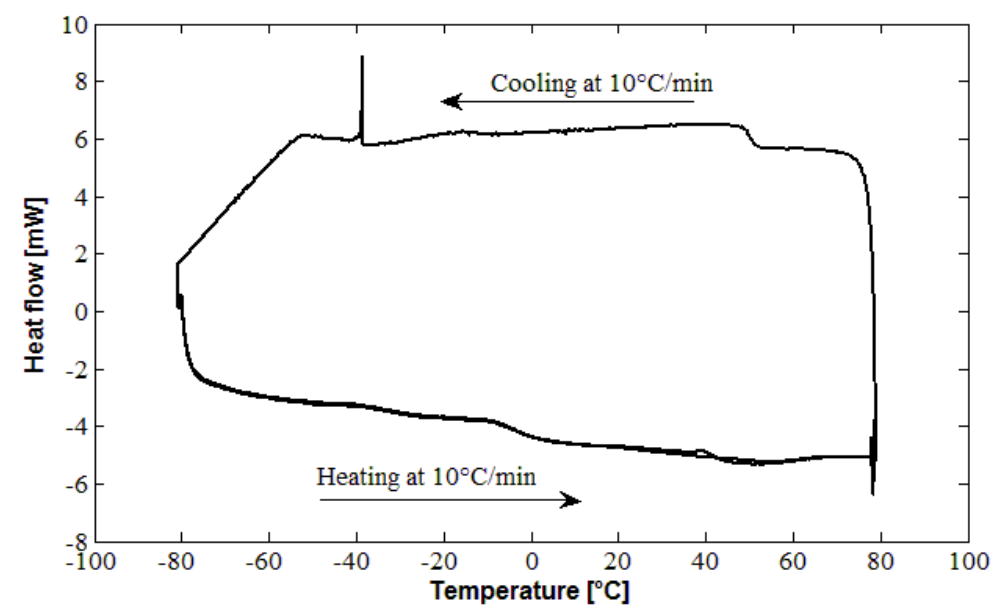

Figure 7. Heat-flow diagram by DSC

\section{AFM after thermal fatigue cycles}

From the above discussion, it can be concluded that bitumen has the tendency to phase separate out structures with predominantly wax. The interface between different phases with different stiffness could generate high stresses due to environmental or mechanical loading. In this study, the sample was exposed to thermal cycles and controlled tensile stresses thus the degraded material properties results into micro-cracks (crazing pattern), as depicted in Figure 8. If this process would continue, these micro-cracks would continue developing and finally form macro-crack.
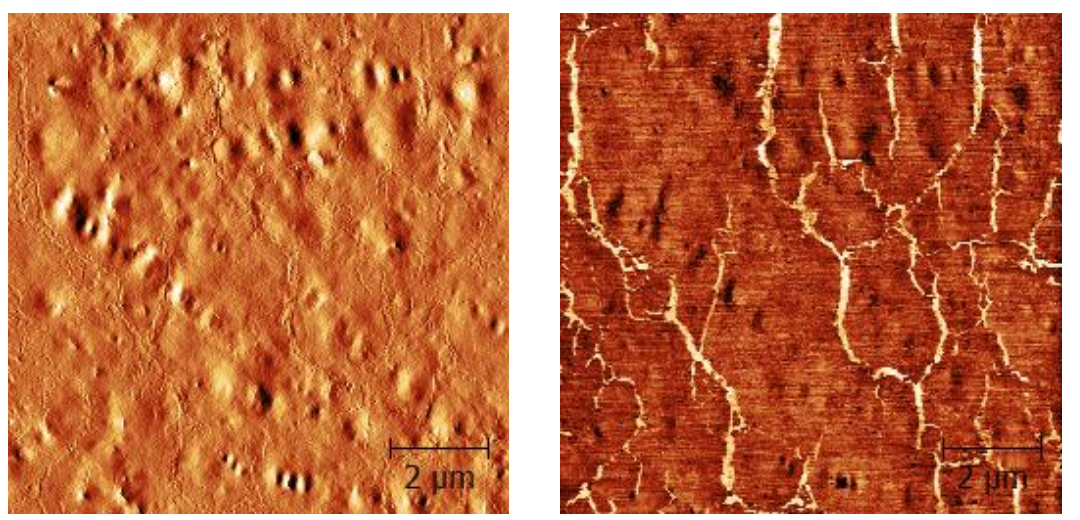

Figure 8. Evidence of micro-crack through AFM scanning: topographic (left) and phase contrast image (right) 


\section{Conclusions}

Atomic force microscopy was used to reveal the microstructures of the bitumen surface which was subjected to both thermal fatigue and controlled stress. As expected, the micro-cracks were found at the interface of the different phases due to stress concentration. This phenomenon proofs bitumen itself may also crack under certain kinetic conditions. In addition, wax crystallization is a very important factor to understand the physico-mechanical behaviour of bitumen as micro structuring is associated with it.

\section{References}

[1] Al-Qadi, I.L., Marwa, M. and Elseifi, M.A. (2005), J. Trans. Res. Record, v. 1919, p. 87-95.

[2] Loeber, L., Sutton, O., Morel, J., Valleton, J.-M. and Muller, G. (1996), J. of Microscopy, v. 182, n. 1, p. 32-39.

[3] Masson, J-F., Leblond, V., Margeson, J. and Bundalo-Perc, S. (2007), J. of Microscopy, vol. 227, n. 3, p. 191-202.

[4] Pauli, A.T., Grimes, R.W., Beemer, A.G., Turner, T.F. and Branthaver, J.F. (2011), Int. J. of Pavement Engineering, v. 12, n. 4, p. 291-309.

[5] Soenen, H. and Vanelstraete, A. (2003), Performance indicators for low temperature cracking, Sixth International RILEM Symposium on Performance Testing and Evaluation of Bituminous Materials, p. 458-464.

[6] Soenen, H., Ekbland, J., Lu, X. and Redelius, P.(2004), Isothermal hardening in bitumen and in asphalt mix, Euroasphalt \& Eurobitumen Congress, report no. 50, p. 1351-63, Vienna.

[7] Edwards, Y. (2005), Influence of waxes on bitumen and asphalt concrete mixture performance, Ph.D. thesis, KTH Royal Institute of Technology, Sweden.

[8] Das, P.K., Tasdemir, Y. and Birgisson, B. (2012), Construction and building Materials, vol. 30, p. 643-649.

[9] Das, P.K., Tasdemir, Y. and Birgisson, B. (2012), Road Materials and Pavement Design, v. 13, n. 1, in press.

[10] Lu, X., Langton, M., Olofsson, P. and Redelius, P. (2005), J. of Materials Science, v. 40, p. 1893-1900.

[11] Lesueur, D., Gerard, J.-F., Claudy, P., Létoffé, J.-M., Planche, J.-P. and Martin, D. (1996). Journal of Rheology, v. 40, n. 5, p. 813-836.

[12] Schmets, A., Kringos, N., pauli, T., Redelius, P. and Scarpas, T. (2010), Int. J. of Pavement Engineering, v. 11, n. 6, p. 555-563.

[13] Pauli, A.T., Branthaver, J.F., Robertson, R.E., Grimes, W. and Eggleston, C.M. (2001), Atomic force microscopy investigation of SHRP asphalts, ACS division of fuel chemistry preprints, v. 46, n. 2, p. 104-110.

[14] Jäger, A., Lackner, R., Eisenmenger-Sittner, Ch. and Blab, R. (2004), Road Materials and Pavement Design, v. 5, p. 9-24.

[15] Lu, X. and Isacsson, U. (2002), Construction and Building Materials, v. 16, n. 1, p. $15-22$. 